\title{
Longitudinal Study on the Influence of Nd:YAG Laser Irradiation on Microleakage Associated with Two Filling Techniques
}

\author{
Maarten Meire, D.D.S., M.Sc., Athina Mavridou, D.D.S., Nikolaas Dewilde, D.D.S., \\ Geert Hommez, D.D.S., M.Sc., Ph.D., and Roeland Jozef Gentil De Moor, D.D.S., M.Sc., Ph.D.
}

\begin{abstract}
Objective: This study investigates the effects of Nd:YAG laser irradiation on apical and coronal seals, when used prior to two root canal filling techniques. Background Data: Limited information exists regarding the effects of morphologic changes to dentin walls following Nd:YAG laser irradiation on the sealing ability of root fillings. Methods: Two hundred forty teeth were analyzed by observing coronal and apical leakage of Indian ink (DL), and 60 were analyzed for through-and-through leakage using the fluid transport model (FTM). The Nd:YAG laser parameters were $1.5 \mathrm{~W}, 100 \mathrm{~mJ}$, and $15 \mathrm{~Hz}$ (four times for $5 \mathrm{~s}$ at $20 \mathrm{~s}$ intervals). Each group consisted of a lased and a nonlased subgroup: each subgroup had root fills done by either cold lateral condensation (CLC) or hybrid condensation (HC). Leakage was assessed after $48 \mathrm{~h}$, and then at 1, 6, and 12 months. The DL group was divided into four groups of 15 teeth for each evaluation point. Through-and-through leakage (L in microliters/ day) was measured for $48 \mathrm{~h}$ under a pressure of $1.2 \mathrm{~atm}$ using FTM, and recorded as $\mathrm{L}=0$ (L1), $0<\mathrm{L} \leq 10$ (L2), or L $>10$ (L3). Results: Apical and coronal dye leakage was observed in all groups. Significant differences $(p<0.05)$ in apical leakage were found between $\mathrm{HC}$ and $\mathrm{HC}+\mathrm{Nd}$ after 1, 6, and 12 months, and between CLC and $\mathrm{CLC}+\mathrm{Nd}$ at 6 and 12 months. No significant differences were found between laser-irradiated and non-laserirradiated groups with FTM. Conclusion: Pulsed Nd:YAG laser irradiation following root canal preparation may reduce apical leakage in association with hybrid gutta-percha condensation.
\end{abstract}

\section{Introduction}

$\mathbf{T}$

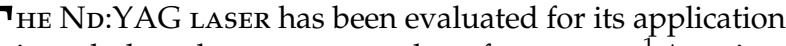
in endodontology over a number of years now. ${ }^{1}$ A variety of studies have shown that direct Nd:YAG laser irradiation on dentin surfaces produces melting and recrystallization which, in turn, causes a reduction in dentin permeability. ${ }^{2-8}$ In view of "dentin melting" and "reduction of the permeability of dentin"4 a number of investigators have attempted to improve the quality of the apical seal and thus reduce apical leakage following apical filling. ${ }^{10-16}$ Apparently, morphological changes following Nd:YAG laser irradiation can influence the marginal sealing of the root canal obturations. Data evaluating coronal leakage of root fillings following laser irradiation of the root canal walls are limited. ${ }^{12}$

In order to prevent the ingress of new pathogens from the oral cavity and maintain the success of the endodontic procedure, adequate three-dimensional obturation and coronal seal, after meticulous cleaning and shaping of the root canal space, are required. ${ }^{17,18}$ In achieving tightly sealed three- dimensional fillings, the hybrid gutta-percha condensation (thermocompaction) is superior to cold lateral condensation (CLC) with respect to apical leakage. ${ }^{19-21}$ However, the overall advantage of hybrid condensation (HC) in lased root canals has not yet been evaluated.

In the present study, we investigated whether Nd:YAG laser-modified dentin root canal walls are associated with improved sealing, particularly when used in association with a warm gutta-percha compaction technique. We evaluated apical and coronal microleakage of filled root canals following root canal treatment, with and without Nd:YAG laser irradiation, and with CLC and hybrid gutta-percha condensation.

\section{Material and Methods}

Tooth selection

This study used 340 extracted human straight singlerooted teeth with mature apices. The distribution of teeth among the experimental groups is shown in Table 1. Carious (limited occlusal and/or interproximal lesions without pulp

Department of Operative Dentistry and Endodontology, Ghent Dental Laser Center, Dental School, Ghent University, Gent, Belgium. 
Table 1. Distribution of the Teeth Among the Experimental Subgroups

\begin{tabular}{|c|c|c|c|c|c|c|c|c|c|c|}
\hline \multirow[b]{2}{*}{ Time interval } & \multicolumn{2}{|c|}{ CLC } & \multicolumn{2}{|c|}{$H C$} & \multicolumn{2}{|c|}{$C L C+N d: Y A G$} & \multicolumn{2}{|c|}{$H C+N d: Y A G$} & \multicolumn{2}{|c|}{ Controls } \\
\hline & $\mathrm{AL}$ & CL & $\mathrm{AL}$ & CL & AL & CL & AL & CL & Positive & Negative \\
\hline \multicolumn{11}{|c|}{ Dye leakage test (Indian ink) } \\
\hline $48 \mathrm{~h}$ & & & & & & & & & 4 & 4 \\
\hline 1 month & & & & & & & & & 4 & 4 \\
\hline 6 months & & & & & & & & & 4 & 4 \\
\hline 12 months & & & & & & & & & 4 & 4 \\
\hline \multicolumn{11}{|c|}{ Fluid transport model } \\
\hline $48 \mathrm{~h}$ & & & & & & & & & 4 & 4 \\
\hline 1 month & & & & & & & & & 4 & 4 \\
\hline 6 month & & & & & & & & & 4 & 4 \\
\hline 12 months & & & & & & & & & 4 & 4 \\
\hline
\end{tabular}

CLC, cold lateral condensation; $\mathrm{HC}$, hybrid condensation; $\mathrm{AL}$, apical leakage; $\mathrm{CL}$, coronal leakage.

exposure) and noncarious teeth were included. All teeth (68 in the dye penetration [DP] groups of $48 \mathrm{~h}, 1,6$ and 12 months, and 68 in the fluid transport model [FTM] subgroup) were stored in $10 \%$ formalin until each experimental subgroup was complete. The preservation time before root canal treatment did not exceed 6 weeks. In order to exclude teeth with multiple root canals, all teeth were radiographed from two angles before root canal treatment. Organic debris was removed by submerging the teeth in $2.5 \%$ sodium hypochlorite for $8 \mathrm{~h}$. Subsequently, they were washed with tap water for $1 \mathrm{~h}$ and stored in saline solution until used. The experimental protocol was approved by the ethical committee of the Ghent University Hospital (Belgium).

\section{Root canal treatment}

Crowns were removed $2 \mathrm{~mm}$ above the cemento-enamel junction using a high-speed fissure bur and water spray. After gross removal of pulp tissues, a size 10 Flexofile (Dentsply Maillefer, Baillaigues, Switzerland) was introduced into the canal until it could be seen in the major apical foramen. The working length was determined by subtracting $1 \mathrm{~mm}$ from this length. The root canals were prepared by means of a crowndown/stepback technique, by one operator. The coronal half of the root canals was preflared with Gates Glidden drills (Dentsply Maillefer) in a larger to smaller sequence (numbers 4-3-2) and the canals were copiously irrigated with $2.5 \%$ sodium hypochlorite solution using a 27-gauge endodontic needle (Monoject, Sherwood Medical, St. Louis, MO, USA). To remove the smear during canal preparation, hand files were covered with the EDTA paste File-Eze (Ultradent Products Inc., South Jordan, UT, USA) before insertion into the root canal. The apical half of the canal was then prepared with the stepback technique up to a master file size 40 . The canals were dried with paper points and the patency of the apical foramen was confirmed with a size 10 Flexofile. The roots were four equal groups of 60 for the investigation of DP, with an additional eight control teeth (four positive and four negative controls), representing the four leakage evaluation times. A second group of 60 teeth with an additional eight control teeth (four positive and four negative controls) was used for leakage evaluation with the FTM. Each group of 60 teeth was then divided into four equal subgroups according to the filling technique used (CLC or $\mathrm{HC}$ ) and whether or not $\mathrm{Nd}$ :YAG lased (Nd) (Table 1). The length of the roots up to the buccal cemento-enamel junction of the experimental groups varied between 16 and $18 \mathrm{~mm}$.

\section{Laser treatment}

In the Nd groups, the root canals were irradiated with a pulsed Nd:YAG laser (FidelisPlus II, Fotona, Ljubljana, Slovenia). A flexible fiber (diameter $0.30 \mathrm{~mm}$ ) was used and inserted into the root canal $1 \mathrm{~mm}$ short of the working length. The laser was then activated and the fiber was moved in a spiral motion along the root canal walls, operating at $1.5 \mathrm{~W}$ and $100 \mathrm{~mJ}$ per pulse with a pulse frequency of $15 \mathrm{~Hz}$. The procedure was repeated four times for $5 \mathrm{~s}$ with a time interval of $20 \mathrm{~s}$. All apical foramina remained patent regardless of Nd:YAG lasing or not (control with a file ISO 15).

\section{Root canal filling: CLC and HC}

A standard size gutta-percha cone (Dentsply Maillefer) that matched the master apical file was fitted to the working length with tug back. Root canal sealer AH 26 (Dentsply Detrey, Konstanz, Germany) was mixed according to the manufacturer's instructions and placed in the canal with the guttapercha to working length. The master cone was then coated again with root canal sealer and gently seated at the working length.

Lateral condensation was carried out using size 20 and 25 accessory gutta-percha cones with endodontic finger spreaders (Dentsply Maillefer) placed in the first instance to within $1 \mathrm{~mm}$ of the working length. The gutta-percha cones coated with sealer were laterally condensed until they could not be introduced more than $3 \mathrm{~mm}$ into the root canal. Following obturation, the gutta-percha was removed from the coronal cavity up to the level of the cemento-enamel junction with a warm instrument (PK Thomas Waxing Instrument, No. PKT-2, Hu Friedy, Leimen, Germany) and vertically condensed with Machtou pluggers (Dentsply Maillefer).

The HC technique consisted of cold lateral gutta-percha condensation of the apical $5 \mathrm{~mm}$ of the root canal (i.e., until spreaders could not be inserted in the gutta-percha of the 
apical $5 \mathrm{~mm}$ ), followed by insertion of a gutta-percha condenser (Dentsply Maillefer) of appropriate size along the gutta cones. The size of the condensers varied between 25 and 40 . The diameter of the condenser allowed insertion into the canal to a depth of at least $8 \mathrm{~mm}$ from the apical preparation. The condenser was then rotated without apical pressure (9000 rpm). After 1-3 s, the gutta-percha was plasticized and the condenser was carried apically up to $3 \mathrm{~mm}$ from the apical stop. The instrument was kept for $1 \mathrm{~s}$ at the desired level and gradually withdrawn, rotating at full speed. Withdrawal speed was determined by the speed of the instrument itself. This obturation technique was a modification of combined lateral condensation and thermatic compaction, as described by Tagger et al..$^{22}$ and De Moor and Hommez. ${ }^{20}$ At the end of the condensation, the gutta-percha was removed from the coronal cavity up to the level of the cemento-enamel junction with a warm instrument and vertically condensed with Machtou pluggers.

After the root filling procedure, a small cotton pellet was sealed in the access cavity of all root-filled teeth using KetacFil (3MEspe, Seefeld, Germany). In roots to be evaluated by FTM a new dry cotton pellet was sealed with Ketac-Fil, after drying the opening cavity with paper points and soft airdrying with the air blower of the dental unit after leakage measurements. The samples were then stored in Vacutainers at $80 \%$ relative humidity for $48 \mathrm{~h}$ at $37^{\circ} \mathrm{C}$ (start of the experiment) and under the same conditions until further measurements at 1, 6, and 12 months. Before storing the teeth, radiographs were taken from the buccal and mesial sides of every tooth.

\section{Control groups}

In addition to the specimens in the experimental groups, 40 root canal-prepared roots were used as controls (Table 1). The canals were not obturated in 20 of the teeth, but the coronal opening as well as the apex was covered with cyanoacrylate cement. All surfaces of the root were then coated with two layers of nail varnish. These were the negative controls. In the remaining 20 teeth, the root canals were not obturated, and the roots were not sealed apically or coronally. These were the positive controls.

\section{Leakage study-Indian ink dye leakage}

At the end of the storage period, the Ketac-Fil restorations and the cotton pellets were removed. Root surfaces were covered with nail varnish. In the dye leakage groups, clear acrylic nail varnish was applied to the entire tooth surface for the negative controls and to all other root surfaces except the apical $2-3 \mathrm{~mm}$ and the coronal opening. The first coat was allowed to dry before a second coat was applied. The teeth were immersed in Indian ink (Pelikan, Hannover, Germany) for $48 \mathrm{~h}$ at $37^{\circ} \mathrm{C}$, after which they were thoroughly washed with running water.

The roots were then grooved longitudinally on the buccal and lingual surfaces, using a small-diameter rotating diamond disk under continuous water cooling. Roots were then carefully fractured and sectioned with a sharp chisel. Care was taken to include the apical foramen in the fracture line. DP was assessed by measuring the amount of dye visible on the gutta-percha filling material or root canal wall. After the gutta-percha filling material was removed with an end- odontic explorer, DP was measured on the canal walls. The DP measurements were compared, and the larger selected as the definitive point of linear dye leakage. To eliminate bias, apical and coronal leakage were measured independently by two evaluators who were unaware of the obturation techniques used. All measurements were obtained using a stereomicroscope (Stemi SR, Zeiss, Oberkochen, Germany) at $\times 6$ magnification, with a calibrated scale ocular.

\section{Leakage study—the FTM}

Ketac-Fil restorations and the cotton pellets were removed prior to leakage studies. The same roots were re-used at the different time intervals; following measurements the opening cavities were air dried, a cotton pellet was placed, and the access cavities were refilled with Ketac-Fil. The roots were then again placed in the Vacutainers until the next assessment of fluid leakage.

The roots were attached to a FTM as described by $\mathrm{Wu}$ et al. ${ }^{23}$ and De Bruyne et al. ${ }^{24}$ Roots were connected at both ends to a plastic tube with Al-Fix Gel (Novatio Belgium n.v., Olen, Belgium) and additionally sealed with Quick-Bond (Novatio). The plastic tubes on either side of the specimen were filled with distilled water, and a standard glass capillary tube was connected to the plastic tube at the outlet side of the specimen. Using a syringe, water was sucked back into the open end of the glass capillary and an air bubble was created. A head space pressure of $1.2 \mathrm{~atm}$ from the inlet side, the coronal side of the tooth, was applied to force the water through the voids along the filling, thus displacing the air bubble in the capillary tube. Microleakage (L in microliters/ day) was measured for $48 \mathrm{~h}$ under a pressure of $1.2 \mathrm{~atm}$ (17 PSI) and recorded as $\mathrm{L}=0$ (L1), $0<\mathrm{L} \leq 10$ (L2), or $\mathrm{L}>10$ (L3). Fluid transport volume was measured by observing movement of the air bubble. The displacement was recorded every $15 \mathrm{~min}$ during the first 6,12 , and at $24 \mathrm{~h}$. The samples were removed from the fluid transport device and stored in the Vacutainers until repeat measurements at 1, 6, and 12 months.

\section{Statistical analysis}

Since the data indicated a nonnormal distribution, dye leakage was assigned using the following categories: 1 ) apical leakage, $0=$ no leakage detected; $1=$ up to $0.5 \mathrm{~mm} ; 2=0.5$ to $1 \mathrm{~mm} ; 3=1$ to $2 \mathrm{~mm} ; 4=>2 \mathrm{~mm}$ leakage; 2 ) coronal leakage, $0=$ no leakage detected; $1=$ up to $1 \mathrm{~mm} ; 2=1$ to $2 \mathrm{~mm} ; 3=2$ to $4 \mathrm{~mm} ; 4=>4 \mathrm{~mm}$ leakage.

Results from both methods were analyzed statistically using nonparametric tests: Kruskal-Wallis and MannWhitney $U$ tests were used to compare leakage following the different techniques and Nd:YAG lasing at $48 \mathrm{~h}$, and 1,6 , and 12 months. The level of significance was set at $0.05(p<0.05)$.

\section{Results}

\section{Dye leakage-apical dye leakage}

Apical dye leakage was observed in all groups (Table 2). Statistically significant differences $(p<0.05)$ were observed between the $\mathrm{HC}$ group and the $\mathrm{HC}+\mathrm{Nd}$ group at 1,6 , and 12 months. Significant differences were also observed between leakage levels in the CLC group and the CLC $+\mathrm{Nd}$ group at 6 and 12 months. 
Table 2. Apical Leakage Scores for the Two Obturation Techniques With and Without Prior ND:YAG LASER IrRAdiation OF THE RoOT CANAL

\begin{tabular}{lcrrrrr}
\hline & & \multicolumn{5}{c}{ Leakage scores $^{\mathrm{a}}$} \\
\cline { 3 - 7 } $\begin{array}{l}\text { Time interval } \\
\text { and group }\end{array}$ & $\begin{array}{c}\text { No. of } \\
\text { specimens }\end{array}$ & 0 & 1 & 2 & 3 & 4 \\
\hline CLC & & & & & & \\
$48 \mathrm{~h}$ & 15 & 12 & 2 & 1 & 0 & 0 \\
1 month & 15 & 10 & 2 & 2 & 1 & 0 \\
6 months & 15 & 9 & 1 & 3 & 2 & 0 \\
12 months & 15 & 8 & 1 & 4 & 2 & 0 \\
HC & & & & & & \\
48 h & 15 & 12 & 2 & 1 & 0 & 0 \\
1 month & 15 & 11 & 2 & 2 & 0 & 0 \\
6 months & 15 & 10 & 2 & 2 & 1 & 0 \\
12 months & 15 & 9 & 3 & 2 & 1 & 0 \\
CLC + Nd:YAG & & & & & & \\
48 h & 15 & 15 & 0 & 0 & 0 & 0 \\
1 month & 15 & 14 & 1 & 0 & 0 & 0 \\
6 months & 15 & 14 & 0 & 1 & 0 & 0 \\
12 months & 15 & 13 & 1 & 2 & 0 & 0 \\
HC Nd:YAG & & & & & & \\
48 h & 15 & 15 & 0 & 0 & 0 & 0 \\
1 month & 15 & 15 & 0 & 0 & 0 & 0 \\
6 months & 15 & 14 & 1 & 0 & 0 & 0 \\
12 months & 15 & 13 & 2 & 0 & 0 & 0 \\
\hline
\end{tabular}

aLeakage scores: 0 , no leakage detected; 1 , up to $0.5 \mathrm{~mm}$; , $0.5-1 \mathrm{~mm} ; 3,1-2 \mathrm{~mm} ; 4,>2 \mathrm{~mm}$ leakage.

\section{Dye leakage - coronal dye leakage}

Coronal leakage was observed in all groups (Table 3). There were no statistically significant differences between the different experimental groups.

\section{Fluid transport model}

No statistically significant differences were found between leakage from laser-irradiated and non-laser-irradiated groups with FTM ( $p>0.05$; Table 4$)$.

\section{Discussion}

Although the use of the Nd:YAG laser as an adjunct in endodontic therapy is well established, a growing number of studies continue to investigate its application. This interest in laser endodontics reflects the relative effectiveness of current cleaning, shaping, and obturation techniques to clean the complex root canal system and the areas that cannot be reached by irrigants and instruments, and to provide the best apical seal. ${ }^{1}$

In this study two different methods were used to assess leakage. Previous studies have emphasized that different leakage evaluation methods have little influence on study results. ${ }^{24-26}$ Dye leakage testing was chosen especially for the assessment of apical leakage, and also provided an additional control for the presence of eventual cracks and fissures in the roots. The FTM chosen for the evaluation of throughand-through leakage had the advantage of re-using the same specimens for sequential leakage measurements. Exposure of the root filling to the water used during the leakage evaluation does not negatively influence the maturation of the sealer used, and hence does not influence additional mea-
Table 3. Coronal Leakage Scores for the Two Obturation Techniques With AND Without PRIOR ND:YAG LASER IRRAdiATION OF THE ROOT CANAL

\begin{tabular}{lcccccc}
\hline & & \multicolumn{5}{c}{ Leakage scores $^{\mathrm{a}}$} \\
\cline { 3 - 7 } $\begin{array}{l}\text { Time interval } \\
\text { and group }\end{array}$ & $\begin{array}{c}\text { No. of } \\
\text { specimens }\end{array}$ & 0 & 1 & 2 & 3 & 4 \\
\hline CLC & & & & & & \\
$\quad 48 \mathrm{~h}$ & 15 & 0 & 1 & 7 & 4 & 3 \\
1 month & 15 & 0 & 1 & 6 & 4 & 4 \\
6 months & 15 & 0 & 0 & 5 & 7 & 3 \\
12 months & 15 & 0 & 0 & 5 & 7 & 3 \\
HC & & & & & & \\
48 h & 15 & 0 & 2 & 9 & 4 & 0 \\
1 month & 15 & 0 & 1 & 9 & 5 & 0 \\
6 months & 15 & 0 & 0 & 6 & 7 & 2 \\
12 months & 15 & 0 & 0 & 6 & 6 & 3 \\
CLC + Nd:YAG & & & & & & \\
48 h & 15 & 0 & 1 & 9 & 5 & 0 \\
1 month & 15 & 0 & 0 & 9 & 4 & 2 \\
6 months & 15 & 0 & 0 & 9 & 3 & 3 \\
12 months & 15 & 0 & 0 & 8 & 4 & 3 \\
HC Nd:YAG & & & & & & \\
48 h & 15 & 0 & 1 & 12 & 2 & 0 \\
1 month & 15 & 0 & 0 & 12 & 3 & 0 \\
6 months & 15 & 0 & 0 & 11 & 4 & 0 \\
12 months & 15 & 0 & 0 & 11 & 4 & 0 \\
\hline
\end{tabular}

aLeakage scores: 0 , no leakage detected; 1 , up to $1 \mathrm{~mm} ; 2,1-2 \mathrm{~mm}$; 3, 2-4 mm; 4, > $4 \mathrm{~mm}$ leakage.

surements. ${ }^{27,28}$ A limitation of the FTM is that it does not give information on the extent of coronal voids.

In the present study, root canals were irrigated with sodium hypochlorite and root canals prepared using an EDTA paste. It was previously shown that although EDTA paste

Table 4. Results of Fluid Transport (Leakage in $\mu \mathrm{L} / \mathrm{D}$ )

\begin{tabular}{lcccc}
\hline & \multicolumn{4}{c}{ No. of samples } \\
\cline { 2 - 5 } & $L=0$ & $0<L \leq 10$ & $L>10$ & Total \\
\hline CLC & & & & \\
48 h & 13 & 2 & 0 & 15 \\
1 month & 13 & 2 & 0 & 15 \\
6 months & 12 & 3 & 0 & 15 \\
12 months & 11 & 4 & 0 & 15 \\
HC & & & & \\
48 h & 15 & 0 & 0 & 15 \\
1 month & 14 & 1 & 0 & 15 \\
6 months & 14 & 1 & 0 & 15 \\
12 months & 14 & 1 & 0 & 15 \\
CLC + Nd:YAG & & & & \\
48 h & 15 & 0 & 0 & 15 \\
1 month & 15 & 0 & 0 & 15 \\
6 months & 14 & 1 & 0 & 15 \\
12 months & 14 & 1 & 0 & 15 \\
HC + Nd:YAG & & & & \\
48 h & 15 & 0 & 0 & 15 \\
1 month & 15 & 0 & 0 & 15 \\
6 months & 15 & 0 & 0 & 15 \\
12 months & 15 & 0 & 0 & 15 \\
\hline
\end{tabular}

L, leakage. 
facilitates root canal preparation, it does not necessarily result in better debris and smear layer removal in the most apical part of the root canal, compared with the sole use of sodium hypochlorite during root canal preparation. ${ }^{29}$ Moreover, it has also been reported that there are no morphological or compositional changes in the root canal walls prepared with hand files, in association with File-Eze, and Nd:YAG lased compared with those prepared without EDTA paste and Nd:YAG lasing. ${ }^{30}$ This contrasts with the use of EDTA as a rinsing solution, with which a final rinse provides effective smear layer removal. ${ }^{29,31}$

These observations are relevant to the present study because a less tight seal has been demonstrated after Nd:YAG lasing when the smear layer is thoroughly removed. ${ }^{13}$ The uniform roughened surface left on the dentin walls after lasing of the smear layer appears to favor cement adhesion, and therefore improves marginal sealing. Regardless of the use of EDTA paste, the apical seal in the lased groups was not negatively affected. These findings are not to be confused with the study of de Moura-Netto et al. ${ }^{16}$ in which Nd:YAG lasing improved apical marginal sealing in EDTA-T rinsed canals, as roots with open apices of an ISO size 40 were evaluated. All studies mentioned so far, however, attribute lower leakage rates to the more evident changes in dentin walls with use of the Nd:YAG, as stated by de Mouro-Netto et al. ${ }^{8}$ The findings of the present study are also in line with those of Gurbuz et al., ${ }^{32}$ which reported no significant differences in root canal wall cleanliness between root canals rinsed with EDTA and those lased with the Nd:YAG laser. Furthermore, the latter study supported that EDTA rinsing is not efficient for cleaning the apical third.

Hybrid gutta-percha condensation has been shown to produce a better apical seal than CLC. ${ }^{19,20}$ The generation of friction heat by condensers results in thermoplasticizing the most apical 3 to $4 \mathrm{~mm}$ gutta-percha, producing better seating of the gutta-percha and a tighter apical seal. In this study, the thermomechanical compaction of the gutta-percha resulted in a better seal in the Nd:YAG lased root canals, as early as 1-month time interval (Table 2). De Moor and Hommez ${ }^{20}$ observed that apical leakage increased with time up to 4 months with guttapercha condensation techniques. These findings are in agreement with those of others, in which leakage increased during the 5 -month evaluation period. ${ }^{33}$ Here an increase in leakage is also found up to the 6-month evaluation period.

$\mathrm{Nd}$ :YAG laser irradiation prior to root canal filling improved the apical seal over several months. This emphasizes the added value of Nd:YAG lasing of root canals where the smear layer has not been completely removed with chelating agents. There is also an ongoing debate over how sodium hypochlorite and EDTA may interfere; a number of studies have shown that the combined use of irrigating solutions decreases the effectiveness of chelating agents and might enhance destruction of the dentinal surface. ${ }^{34}$ Furthermore, combined use of irrigating solutions might have negative effects on the bond strength of adhesive cement to root canal dentin. ${ }^{35,36}$ A major advantage of the Nd:YAG laser technique is therefore its cleaning efficacy and low tendency to produce mineral loss from the root canal dentin. ${ }^{32}$

\section{Conclusion}

Pulsed Nd:YAG laser irradiation following root canal preparation reduces apical leakage in teeth with maturated root fillings, especially in combination with hybrid guttapercha condensation.

$\mathrm{Nd}$ :YAG lasing of root canal walls covered by a smear layer also resulted in an improved root canal seal.

\section{Disclosure Statement}

No competing financial interests exist.

\section{References}

1. Meire, M., and De Moor, R.J.G. (2007). Lasers in endodontics: laser disinfection, an added value? Endodont Pract Today 1, 159-172.

2. Dederich, D.N., Zakariasen, K.L., and Tulip, J. (1984). Scanning electron microscopic analysis of canal wall dentin following neodymium-yttrium-aluminium-garnet laser irradiation. J. Endod. 10, 428-431.

3. Levy, G. (1992). Cleaning and shaping the rot canal with a Nd:YAG laser beam: a comparative study. I. Endod. 18, 123127.

4. Stabholz, A., Khayat, A., Weeks, D.A., Neev, J., and Torabinejad, M. (1992). Scanning electron microscopic study of the apical dentine surfaces lased with Nd:YAG laser following apicectomy and retrofill. Int. Endod. J. 26, 288-291.

5. Miserendino, L.J., Levy, G.C., and Riziou, I.M. (1995). Effects of Nd:YAG laser on the permeability of root canal wall dentin. J. Endod. 21, 83-87.

6. Anic, I., Tachibana, H., Matsumoto, K. and Qi, P. (1996). Permeability, morphologic and temperature changes of canal dentine walls induced by Nd:YAG, $\mathrm{CO}_{2}$ and argon lasers. Int. Endod. J. 1, 13-22.

7. Altamura, C., Majori, M., Bedini, R., and Filippini, P. (2003). Evaluation of Nd:YAG laser effects on root canal walls. J. Oral Laser Appl. 2, 67-72.

8. de Moura-Netto, C., Antonio, M.P.S., Moura, A.A.M., Davidowivcz, H., Carvalho, C.F., and Lage-Marques, J.L. (2005). Morphologic analysis of dentin surfaces on apical third after Nd:YAG laser and diode laser irradiation. Photomed. Laser Surg. 1, 117.

9. Takeda, F.H., Harashima, T., Kimura, Y., and Matsumoto, K. (1998). Comparative study about the removal of smear layer by three types of laser devices. J. Clin. Laser Med. Surg. 16, $117-122$.

10. Kimura, Y., Yamazaki, R., Goya, C., Tomita, Y., Yokoyama, K., and Matsumoto, K. (1999). A comparative study on the effects of three types of laser irradiation at the apical stop and apical leakage after obturation. J. Clin. Laser Med. Surg. 17, 261-266.

11. Goya, C., Yamazaki, R., Tomita, Y., Kimura, Y., and Matsumoto, K. (2000). Effects of pulsed Nd:YAG laser irradiation on smear layer at the apical stop and apical leakage after obturation. Int. Endod. J. 33, 266-271.

12. Park, D.S., Lee, H.J., and Yoo, H.M. (2001). Effect of Nd-YAG laser irradiation on the apical leakage of obturated root canals: an electrochemical study. Int. Endod. J. 34, 318-321.

13. Carvalho, C.A.T., Valera, M.C., Gown-Soars, S., and De Paulo Eduardo, C. (2002). Effects of Nd:YAG and Er:YAG lasers on the sealing of root canal fillings. J. Clin. Laser Med. Surg. 20, 215-219.

14. Depraet, F.J.H.W., De Bruyne, M.A.A., and De Moor, R.J.G. (2005). The sealing ability of an epoxy resin root canal sealer after Nd:YAG laser irradiation of the root canal. Int. Endod. J. 38, 302-309.

15. Sousa-Neto, M.D., Silva Coelho, F.I., Marchesan, M.A., Alfredo, E., and Silva-Sousa,Y.T. (2007). Ex vivo study of the 
adhesion of an epoxy-based sealer to human dentine submitted to irradiation with Er:YAG and Nd:YAG lasers. Int. Endod. J. 38, 866-870.

16. de Moura-Netto, C., Carvalho, C.F., Moura, A.A.M., Davidowicz, H., and Antoniazzi, J.H. (2007). Influence of Nd: YAG and diode laser irradiation on apical sealing when associated with AH Plus and EndoRez endodontic cements. Photomed. Laser Surg. 5, 413-417.

17. Hommez, G.M.G, Coppens, C., and De Moor R.J.G. (2002). Periapical health related to the quality of coronal restorations and root fillings. Int. Endod. J. 35, 680-689.

18. Schilder, H. (2006). Filling root canals in three dimensions. J. Endod. 32, 281-290.

19. De Moor, R.J.G., and Martens, L.C. (1999). Apical microleakage after lateral condensation, hybrid gutta-percha condensation and Soft-Core obturation: an in vitro evaluation. Endod. Dent. Traumatol. 15, 239-243.

20. De Moor, R.J.G., and Hommez, G.M.G. (2002). The sealing ability of an epoxy resin root canal sealer used with five guttapercha obturation techniques. Int. Endod. J. 35, 200-208.

21. De Moor, R.J.G., and De Bruyne, M.A.A. (2004). The long term sealing ability of $\mathrm{AH} 26$ and $\mathrm{AH}$ Plus used with three guttapercha obturation techniques. Quint. Int. 35, 326-331.

22. Tagger, M., Tamse, A., Katz, A., and Korzen, B.H. (1984). Evaluation of the apical seal produced by a hybrid root canal filling method, combining lateral condensation and thermatic compaction. J. Endod. 10, 299-303.

23. Wu M.-K., De Gee A.J., Wesselink, P.R., and Moorer, W.R. (1993). Fluid transport and bacterial penetration along root fillings. Int. Endod. J. 26, 203-208.

24. De Bruyne, M.A.A., De Bruyne, R.J.E., Rosiers, L., and De Moor, R.J.G. (2005). Longitudinal study on microleakage of three root-end filling materials by fluid transport method and by capillary flow porometry. Int. Endod. J. 38, 129-136.

25. Wu, M-K., and Wesselink, P.R. (1993). Endodontic leakage studies reconsidered. Part I. Methodology, application and relevance. Int. Endod. J. 26, 37-43.

26. Pommel, L., Jacquot, B., and Camps, J. (2001). Lack of correlation among three methods for evaluation of apical leakage. J. Endod. 27, 347-350.

27. Wu, M.-K. (1993). In vitro leakage determination for endodontic research. Ph.D. thesis. Amsterdam, The Netherlands: University of Amsterdam.
28. De Bruyne, M.A.A. (2006). Ultrasonic root-end preparation and sealing ability of conventionally-setting glass ionomer cement in surgical endodontics. Ph.D. thesis. Gent, Belgium: Academia Press, Ghent University, Dental School.

29. Torbeyns, D.R.H., De Bruyne, M.A.A., and De Moor, R.J.G. (2007). A SEM study of smear layer removal in association with $\mathrm{NaOCl}$ and EDTA formulations. Int. Endod. J. 40, 991 R2.38.

30. Mavridou, A. (2008). A microRaman and scanning electron microscopic evaluation of root canal walls exposed to Nd:YAG and KTP laser. Master thesis. Gent, Belgium: Ghent University, Dental School.

31. Niu, W., Yoshioka, T, Kobayashi, C., and Suda, H. (2002). A scanning electron microscopic study of dentinal erosion by final irrigation with EDTA and $\mathrm{NaOCl}$ solutions. Int. Endod. J. 35, 934-939.

32. Gurbuz, T., Ozdemir, Y., Kara, N., Zehir, C., and Kurudirek, M. (2008). Evaluation of root canal dentin after Nd:YAG laser irradiation and treatment with five different irrigation solutions: a preliminary study. J. Endod. 34, 318-321.

33. Gurney, B.F., Best, E.J., and Gervasio, G. (1971). Physical measurements on gutta-percha. Oral Surg. Oral Pathol. Oral Med. 32, 260-270.

34. Dogan, H., and Qalt, S. (2001). Effects of chelating agents and sodium hypochlorite on mineral content of root dentin. J. Endod. 27, 578-580.

35. Ari, H., and Erdemir, A. (2005). Effects of irrigation solutions on mineral content of root canal dentin using ICP-AES technique. J. Endod. 31, 187-189.

36. Erdemir, A, Ari, H., Güngünes, H., and Belli, S. (2004). Effect of medications for root canal treatment on bonding to root canal dentin. J. Endod. 30, 113-116.

Address correspondence to: Roeland De Moor, D.D.S., M.Sc., Ph.D. Department of Operative Dentistry and Endodontology Dental School Ghent University, Ghent University Hospital De Pintelaan 185/P8 B-9000 Gent Belgium

E-mail: roeland.demoor@ugent.be 


\section{This article has been cited by:}

1. Rory E. Mortman. 2011. Technologic Advances in Endodontics. Dental Clinics of North America 55:3, 461-480. [CrossRef]

2. Asude Yilmaz, Musa \#amil Akyil , Bilal Hologlu . 2011. The Effect of Metal Primer Application and Nd:YAG Laser Irradiation on the Shear-Bond Strength Between Polymethyl Methacrylate and Cobalt-Chromium AlloyThe Effect of Metal Primer Application and Nd:YAG Laser Irradiation on the Shear-Bond Strength Between Polymethyl Methacrylate and CobaltChromium Alloy. Photomedicine and Laser Surgery 29:1, 39-45. [Abstract] [Full Text] [PDF] [PDF Plus]

3. Rafaël Michiels, Tom Edgard Maria Vergauwen, Athina Mavridou, Maarten Meire , Mieke De Bruyne , Roeland Jozef Gentil De Moor . 2010. Investigation of Coronal Leakage of Root Fillings After Smear-Layer Removal with EDTA or Nd:YAG Lasing Through Capillary-Flow PorometryInvestigation of Coronal Leakage of Root Fillings After Smear-Layer Removal with EDTA or Nd:YAG Lasing Through Capillary-Flow Porometry. Photomedicine and Laser Surgery 28:S2, S-43-S-50. [Abstract] [Full Text] [PDF] [PDF Plus] 